\title{
Growth Retardation, Duodenal Lesions, and Aberrant lleum Architecture in Triple Null Mice Lacking EGF, Amphiregulin, and TGF- $\alpha$
}

\author{
KELLY L. TROYER, $*$ NOREEN C. LUETTEKE, ${ }^{\ddagger}, \AA$ MARIAN L. SAXON, ${ }^{\ddagger}$ TING HU QIU, ${ }^{\ddagger}$ CORY J. XIAN, $\$$ \\ and DAVID C. LEE ${ }^{\dagger}, \|$ \\ Departments of "Biochemistry \& Biophysics and * Microbiology \& Immunology, ${ }^{*}$ UNC Lineberger Comprehensive Cancer Center, University of \\ North Carolina School of Medicine, Chapel Hill, North Carolina; \$Child Health Research Institute, and Flinders University Department of \\ Human Physiology, Adelaide, Australia; and $\rrbracket^{\mathbb{H}} \mathrm{H}$. Lee Moffitt Cancer Center, University of South Florida, Tampa, Florida
}

Background \& Aims: Mice lacking epidermal growth factor (EGF), transforming growth factor $\alpha$, and amphiregulin were used to identify roles for these EGF receptor (EGF-R) ligands in gastrointestinal development and mucosal integrity. Methods: Gastrointestinal tract development was examined in knockout mice and correlated with expression of EGF-R protein and EGF family members throughout the gut. Crossfostering experiments addressed roles of maternal- and neonatal-derived ligands in pup growth and intestinal development. Cysteamineinduced ulceration in $\mathrm{EGF}^{-/-}$mice was used to examine its role in mucosal cytoprotection. Results: Neonatal mice lacking all 3 ligands were growth retarded, even when reared by wild-type dams; conversely, lack of maternal ligands transiently impaired wild-type pup growth. Triple null neonates displayed spontaneous duodenal lesions, and ileal villi were truncated and fragile with reduced cellular proliferation in the crypts. However, maturation of digestive enzymes was unaffected. Adult $\mathrm{EGF}^{-/-}$mice displayed more severe lesions in response to cysteamine treatment compared with wild-type counterparts, although triple null mice were not more susceptible to dextran sulfate sodium-induced colitis, suggesting a differential role for these ligands in the injury response. Conclusions: EGF-R ligands are required for development and mucosal maintenance in mouse small intestine. Both maternal and neonatal sources of growth factors are required for optimal pup growth.

G pidermal growth factor receptor (EGF-R) (also known C as ERB1) and its ligands are implicated in development and homeostasis of the gastrointestinal tract. The 6 activating ligands include: epidermal growth factor (EGF), transforming growth factor (TGF)- $\alpha$, amphiregulin (AR), heparin-binding EGF (HB-EGF), betacellulin (BTC), and epiregulin (EPR). ${ }^{1}$ Ligand binding induces EGF-R dimerization, activates the intrinsic tyrosine kinase, and couples the receptor to downstream signaling pathways controlling cell proliferation, differ- entiation, survival, or motility. EGF-R functions as part of a network, forming heterodimers with 3 related proteins, ERBB2/C-neu, ERBB3, and ERBB4, and 3 of the EGF-R ligands, HB-EGF, BTC, and EPR, also directly bind ERBB 4. 2,3 Although EGF, TGF- $\alpha$, and AR only bind EGF-R, they can nevertheless produce quantitatively or qualitatively distinct biological responses. ${ }^{4,5}$

Components of the EGF-R signaling system are expressed in the gut, and at least some of the ligands are present in saliva and milk. 6,7 Suggestive of important roles in gut ontogeny, exogenous EGF or TGF- $\alpha$ are strong inducers of mucosal growth, ${ }^{8-10}$ and intestinal hyperplasia was observed in transgenic mice overexpressing TGF- $\alpha .{ }^{11}$ Additionally, numerous reports raise the possibility that EGF-R signaling might influence intestinal maturation in rodents, ${ }^{12}$ although it remains uncertain whether effects of EGF on disaccharidase activities are physiologic. In the mature gut, activated EGF-R may function in repair because numerous reports show that exogenous EGF or TGF- $\alpha$ ameliorates mucosal damage in chemical models. ${ }^{13}$ EGF, TGF- $\alpha$, and EGF-R are each induced at sites of gastrointestinal wounding ${ }^{14}$ and may regulate trefoil expression as part of the healing response. ${ }^{15}$ Finally, the EGF-R system may have other, nonmitogenic effects on gastrointestinal physiology that include regulation of gastric acid and mucus secretion, as well as control of intestinal blood flow and motility. ${ }^{13}$

Surprisingly, mice lacking TGF- $\alpha$, EGF, or AR individually display no overt gastrointestinal phenotypes,

\footnotetext{
Abbreviations used in this paper: AR, amphiregulin; BTC, betacellulin; cDNA, complementary DNA; DSS, dextran sulfate sodium; EGF, epidermal growth factor; EGF-R, EGF receptor; EPR, epiregulin; HBEGF, heparin-binding EGF; ITF, intestinal trefoil factor; mRNA, messenger RNA; RT-PCR, reverse-transcription polymerase chain reaction; TGF, transforming growth factor.

(C) 2001 by the American Gastroenterological Association 0016-5085/01/ \$35.00 doi:10.1053/gast.2001.25478
} 
perhaps because of the redundancy of EGF-R ligands. ${ }^{16-18}$ In addition, multiple lines of mice lacking EGF-R showed only mild gastrointestinal abnormalities at late gestation. ${ }^{19,20}$ However, on a Swiss-Webster background, loss of EGF-R resulted in a dramatic intestinal phenotype in neonates that was characterized by a hemorrhagic intestine with short, sparse villi. ${ }^{21}$ Despite having no overt gut phenotype, mice lacking TGF- $\alpha$ or harboring a kinase-defective EGF-R (Waved-2) ${ }^{22}$ were more sensitive to dextran sulfate-induced colonic injury ${ }^{23}$ and showed impaired adaptation after small bowel resection, ${ }^{24}$ respectively. These results lend further support to roles for EGF-R and its ligands in aspects of gut development and homeostasis.

In the present report, we examine gastrointestinal defects in triple null mice that simultaneously lack AR, EGF, and TGF- $\alpha$ (designated aaeett mice $\left.{ }^{18}\right)$. These mice are growth retarded, but viable and fertile. We report here that the duodenum of aaeett weanling mice is prone to spontaneous ulceration, likely related to decreased mucin production, and that in the ileum, villi seem truncated and fragile concomitant with decreased DNA synthesis in the crypts. We further show that adult mice lacking only EGF develop more severe duodenal lesions in response to cysteamine treatment than their wild-type counterparts. These findings provide important genetic evidence of roles for EGF, TGF- $\alpha$, and AR in aspects of gastrointestinal development and homeostasis.

\section{Materials and Methods}

\section{Animals}

All mice ${ }^{18}$ were maintained on a mixed genetic background of C57BL/6 and 129/SvJ strains through crosses of like genotype mice. Mice had free access to water and Purina (St. Louis, MO) lab chow in a climate controlled environment and were killed by $\mathrm{CO}_{2}$ asphyxiation, in accordance with Institutional Animal Care and Use Committee procedures. Tissues were fixed in $10 \%$ buffered formalin and sectioned by the UNC Histopathology Core Facility.

Fasted, 4-6-month-old adult female mice were administered cysteamine by gavage at a dose of $500 \mathrm{mg} / \mathrm{kg}$ and killed 24 ( $\mathrm{n}=32$ wild-type, 33 AAeeTT mice) or 48 hours $(\mathrm{n}=25$ wild-type, 24 AAeeTT mice) later. Duodenal lesions were observed by stereomicroscopy of opened mucosal surface and scored as indicated in a blind comparison. Histologic sections encompassing lesions were used to reevaluate scores.

\section{Molecular Analyses}

RNA was isolated from fresh tissues with Trizol (BRL, Grand Island, NY). Low-cycle number reverse-transcription polymerase chain reaction (RT-PCR) assays (20-25 cycles) used the following primers: TGF- $\alpha,(F)$ GTGGCTGCAGCACCCTGCGCT, $(R)$ GATCAGCACACAGGTGATAAT-
GAGGAC; EGF, (F) GCAACTCCGTCCGGGCGAGGA, $(R)$ GAAGATGACTGTGGTCCCGGG; AR, $(F)$ GCCATTATGCAGCTGCTTTGGAGC, $(R)$ TGTTTTTCTTGGGCTTAATCACCT; HB-EGF, (F) ATGAAGCTGCTGCCGTCGGTGATGCTGA, (R) GGTATCTGCACTCCCCGTGGATGC; BTC, $(F)$ ATGGACCCAACAGCCCCGGGTAGCAGTG, $(R)$ TCGCTCACACCGAGCCCCAAAGTA; EPR $(F)$ GACCTGTGGCAGCACTGGGTCCCC, $(R)$ ATCATGCATCCCAGGAGAATCCGA; EF-1 $\alpha,(F)$ AGCGTGGTATCACTATTGA, $(R)$ ATCTTTGCGGGTGACTTTC; intrinsic factor, $(F)$ CATGGAGAACTCGGTGACTG, $(R)$ CTCCTGTGTCCACATTGAAG; and carbonic anhydrase, $(F)$ ATGGAGCAAGCTGTATCCCATTGC, $(R)$ GAGCCATGGTCGTTTCAGTTGC. Ligand complementary DNA (cDNA) probes used were described $^{25}$; control probes were radiolabeled internal 50-base oligonucleotides. For Northern blots, a mouse sucrase-isomaltase probe was derived by PCR amplification of the $3^{\prime}$ UT region (GenBank \#AI641904) using primers (F) CGTTGGATGCAAGTTGGAGC and (R) GGTAGTCAAACCATCGAGCC, and verified by sequencing. A rat lactase ${ }^{26} \mathrm{CDNA}$ was kindly provided by Dr. Robert Montgomery, Tufts University. Western blots were performed as described ${ }^{25}$ after determining protein concentrations using the BioRad Protein Assay (BioRad, Hercules, CA). The intestinal trefoil factor (ITF) antibody was generously provided by Dr. Andrew S. Giraud, University of Melbourne.

\section{Statistical Analysis}

Results are expressed as mean \pm SEM. Crossfostering data were analyzed by random coefficient modeling, using PROC MIXED (SAS software version 8.0, Cary, NC); other data were analyzed by the Wilcoxon signed-rank, the Fisher exact, and $\chi^{2}$ tests $(P<0.05$ considered significant). All statistical analyses were performed by the UNC Lineberger Comprehensive Cancer Center Biostatistics Core.

\section{Results}

\section{Mice Lacking AR, EGF, and TGF- $\alpha$ Are Growth Retarded}

We previously noted that postnatal growth of triple null (aaeett) pups was impaired relative to wildtype counterparts. ${ }^{18} \mathrm{We}$ further showed that growth of triple hemizygous, (AaEeTt) pups born to aaeett dams was retarded, but not as severely, suggesting a role for both maternal- and neonatal-derived growth factors. To corroborate this, we performed crossfostering experiments in which aaeett pups were nursed by wild-type dams and wild-type pups were reared by aaeett dams. These experiments distinguish between potential contributions of reduced milk quantity and/or quality caused by impaired aaeett mammary gland development vs. elimination of neonatal growth factors. We selectively used aaeett dams that successfully weaned their first litter (the majority do not); these dams had approximately 
$50 \%$ ductal development when killed upon litter weaning. This strategy resulted in a higher frequency of pup survival than occurs with random aaeett dams ( $>90 \%$ vs. $\left.25 \%{ }^{18}\right)$. For crossfostering, dams were provided with completely new, mixed litters composed of 3 pups of each genotype. Small litter size encouraged equal access to milk, and mixed litters controlled for nurturing behavior.

Although not apparent in Figure 1, the average weights of newborn aaeett pups were slightly reduced relative to that of wild-type pups $(1.33 \pm 0.02$ vs. $1.43 \pm 0.01 \mathrm{~g})$, a statistically significant $(P=0.0001)$ difference. However, this difference became more exaggerated, resulting in a $40 \%$ reduction in the body weight of aaeett pups by 3 weeks, the time of weaning. Significantly, aaeett body weight was only partially restored when these pups were nursed by wild-type dams; conversely, wild-type pups fed aaeett milk had reduced body weights, relative to the wild-type control pups. Thus, consistent with our earlier results, optimal postnatal growth required both maternal and pup sources of ligands; loss of either was deleterious. The difference in body weights diminished with age: by 7 weeks, aaeett weights were $15 \%$ reduced relative to wild-type, and the influence of the dam's genotype was no longer apparent (data not shown).

\section{Triple Null Mice Display Duodenal Lesions}

Because a large literature implicates both milkborne and endogenous EGF family members in gut

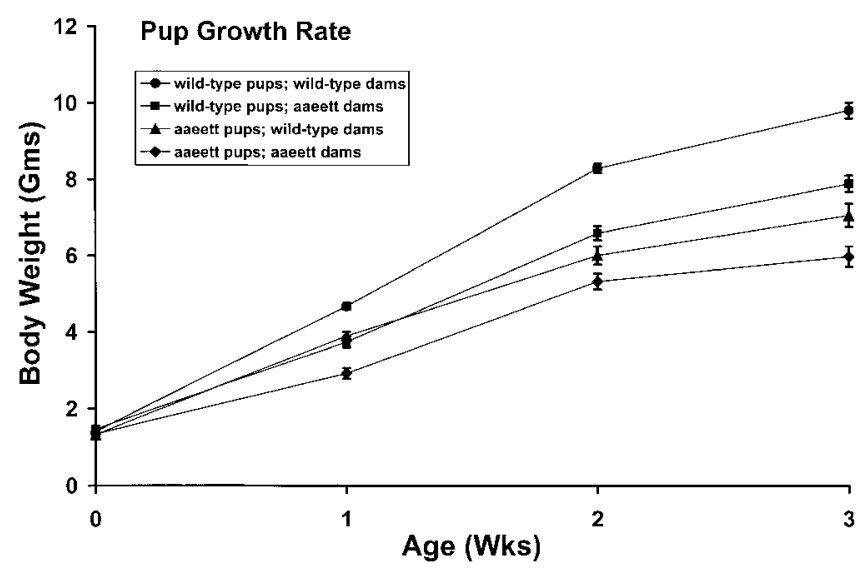

Figure 1. Growth rates of crossfostered pups. Newborn wild-type ( $\mathrm{n}=$ $42)$ and aaeett $(n=42)$ pups were weighed and distributed equally to wild-type $(n=7)$ and aaeett $(n=7)$ biparous dams, preserving a constant litter size of 6 pups. Pup body weight was subsequently determined at weeks 1,2 , and 3 . Survival at week 3 was $81 \%$ for aaeett pups nursed by wild-type dams, $100 \%$ for wild-type pups reared by aaeett dams, and $93 \%$ for each of the 2 remaining groups. Although not apparent, the average aaeett birth weight was $7 \%$ reduced relative to that of wild-type pups, a small but statistically significant difference. Differences between the control $(\mathbf{0} ;)$ and crossfostered $(\boldsymbol{\square} ; \mathbf{\Delta})$ groups were statistically significant $(\alpha<0.05)$, but the difference between the experimental, crossfostered groups was not. development, and one line of EGF-R null mice displayed severe intestinal disintegration, ${ }^{21}$ we examined aaeett mice for gastrointestinal abnormalities as a possible explanation for the observed growth retardation. Although most compartments of the aaeett gut displayed normal morphology, notable defects were observed in the duodenum and ileum. In the duodenum, spontaneous lesions affecting mucosal integrity were observed in $\sim 50 \%$ of samples. These varied from mild erosions and ulcerations (Figure $2 A, a-c$ ) to occasional severe perforations (Figure $2 A$, a and $d$ ).

It has been suggested that EGF family members are involved in the regulation of cytoprotective mucus secretion in the gastrointestinal tract, ${ }^{13}$ and TGF- $\alpha$ treatment increases mucin levels in the stomach. ${ }^{27}$ To evaluate mucin production, we compared Mayer's mucicarmine staining of wild-type and aaeett duodenum. Most aaeett villous goblet cells stained less robustly with mucicarmine, suggesting they are producing lower amounts of mucin (Figure 2B; compare arrowheads). Additionally, decreased cytoprotection in the aaeett duodenum is likely related to the fact that the number of villous goblet cells was decreased $32 \%$ relative to wildtype villi ( $\mathrm{n}=15$ well-oriented villi/mouse, 3 mice/ genotype). Neither spontaneous lesions nor altered mucin staining were observed in mice lacking any pair-wise combination of AR, EGF, and TGF- $\alpha$ (data not shown), indicating either that all 3 contribute to cytoprotection normally, or that 1 or more serve this function in response to loss of physiologically relevant ligands.

Finally, because EGF-R signaling may also regulate the production of trefoils, a peptide family critical for mucosal defense and ulcer healing, ${ }^{28-31}$ and because trefoils and mucins may be coregulated, ${ }^{32}$ we assessed trefoil production in triple null mice. Western blots using anti-ITF ${ }^{28}$ revealed variably reduced levels of ITF in proximal, but not distal, small intestine of 3-week-old aaeett mice compared with wild-type counterparts (data not shown). However, because ITF is prominently produced by goblet cells, ${ }^{33}$ this reduction may largely reflect the diminished numbers of goblet cells observed in triple null duodenum. Indeed, remaining goblet cells in weanling aaeett small intestine stained positively for ITF (data not shown). Reductions in trefoil production, combined with diminished mucin levels, could account for the increased incidence of spontaneous lesions in the aaeett duodenum.

\section{Ileum of aaeett Mice Exhibits Aberrant Architecture}

In the ileum of 3-week-old mice, we observed altered architecture, owing in part to hypocellularity. In 
contrast to the smooth finger-like projections of wildtype ileum (Figure 3A, a), aaeett villi (Figure 3A, c) were dramatically stunted. They also appeared fragile with jagged or sheared tips (Figure 3A, d; inset), and fractured villi were often observed in the lumen. When measured at $40 \times$ magnification, the average villus height in aaeett ileum was reduced $47 \%$ relative to wild-type (218.26 \pm 2.73 vs. $116.09 \pm 2.48 \mu \mathrm{m} ; \mathrm{n}=20$ well-oriented villi/mouse, 5 mice/genotype). We also note that the muscularis mucosa layer of aaeett ileum was frequently reduced in thickness compared with the wild-type counterpart (Figure $3 A, \mathrm{~b}$ and $\mathrm{d}$ ). The significance of this observation, and specifically whether it reflects general hypoplasia in this tissue or instead indicates that the 3 EGF-R ligands promote growth of intestinal smooth muscle cells, is unclear. These defects were transient in nature because ileum from 5 -week-old aaeett mice appeared histologically normal with only a slight $(\sim 11 \%)$ reduction in villus height (data not shown). Similar defects were observed in 3 of 9 aaeett pups reared by wild-type dams, whereas ileum from 12 wild-type pups nursed by aaeett dams appeared normal. Consistent with the morphologic results, the weight/length ratio of the small intestine was reduced $37 \%$ in aaeett pups nursed by like dams, improved modestly when they were fed wild-type milk, but was unaffected in wild-type pups regardless of the milk source (Table 1). Collectively, these various findings reveal a requirement for neonatally derived EGF-R ligands in establishment or maintenance of ileum architecture.

The hypocellularity of aaeett ileum could be caused by decreased cell proliferation or increased apoptosis and/or cell sloughing. As assessed by BrdU incorporation, $37.9 \% \pm 2.8 \%$ of crypt epithelial cells were proliferating in 3 -week-old wild-type ileum, a value in close agreement with a published report, ${ }^{34}$ but only $22 \% \pm$ $0.3 \%$ of cells were proliferating in counterpart aaeett ileum (Figure 3B). No differences in BrdU staining were observed in the aaeett duodenum or jejunum, consistent with the normal appearance of villi in these segments. Apoptosis, as measured by the TUNEL assay, was not appreciable in the ileum of either wild-type or aaeett mice at this age. Hence, reduced cell proliferation likely accounts for the observed hypocellularity, although increased cell sloughing may contribute.

\section{Functional Maturation of the Small Intestine Is Not Affected in Triple Null Mice}

Coordinated with weaning, the small intestine undergoes a critical functional switch from expression of digestive enzymes required for a milk-based diet to adult enzymes necessary for the digestion of solid food. EGF-R ligands have been implicated in this process of intestinal maturation. ${ }^{12}$ Thus, we compared expression of lactase and sucrase-isomaltase genes in the jejunum because it expresses high levels of these enzymes and its architecture was normal in aaeett mice. Northern blots showed that peak levels of messenger RNAs (mRNAs) encoding lactase and sucrase-isomaltase were similar in wild-type and aaeett mice (Figure 4). Moreover, lactase mRNA levels were similarly reduced in jejunum of both genotypes at 3 weeks of age, whereas sucrase-isomaltase mRNA levels similarly increased from barely detectable levels at 2 weeks of age (data not shown), suggesting that both the timing and extent of maturation was unaffected by loss of the 3 EGF-R ligands.

\section{EGF-R Ligands Are Broadly Expressed in the Gut}

Although the defects observed in the aaeett duodenum and ileum resemble aspects of the phenotypes produced by EGF-R deficiency in an outbred mouse line, ${ }^{21}$ they are focal and less severe, consistent with expression of remaining receptor ligands in the gut. Expression of certain ligands, principally EGF and TGF- $\alpha$, has been studied in human and rodent gastrointestinal tracts, but the family has not been comprehensively surveyed in mice. To analyze expression of the 6 ligands throughout the gut of multiple 3-week-old animals, we used a semiquantitative RT-PCR assay, ${ }^{25}$ normalizing the input cDNA to yield comparable amplification of elongation factor $1 \alpha$ transcripts. ${ }^{35}$ Validating the assay, intrinsic factor ${ }^{36}$ and carbonic anhydrase $1^{37}$ transcripts were highly localized to samples respectively derived from stomach and colon as expected (Figure 5). In marked contrast to intrinsic factor and carbonic anhydrase 1 mRNAs, transcripts for all 6 EGF-R ligands were found throughout the gut, although some preferential expression was observed. For example, although levels of TGF- $\alpha$ and AR transcripts were comparable in most tissues, EGF, BTC, and EPR mRNAs were enriched in stomach, and HB-EGF transcripts were elevated in the small intestine (Figure 5). The finding that HB-EGF, BTC, and EPR are broadly expressed in the gut provides a possible explanation for the milder phenotype of aaeett vs. EGF-R null mice but does not account for the focal nature of aaeett defects.

In addition to comparing ligand expression, we evaluated EGF-R protein levels in wild-type and aaeett mice over the course of postnatal gut development, including the early phase of crypt organization and villus extension. At the times indicated in Figure 6, EGF-R protein was immunoprecipitated from independent samples of proximal or distal small intestine, or colon, and then immu- 

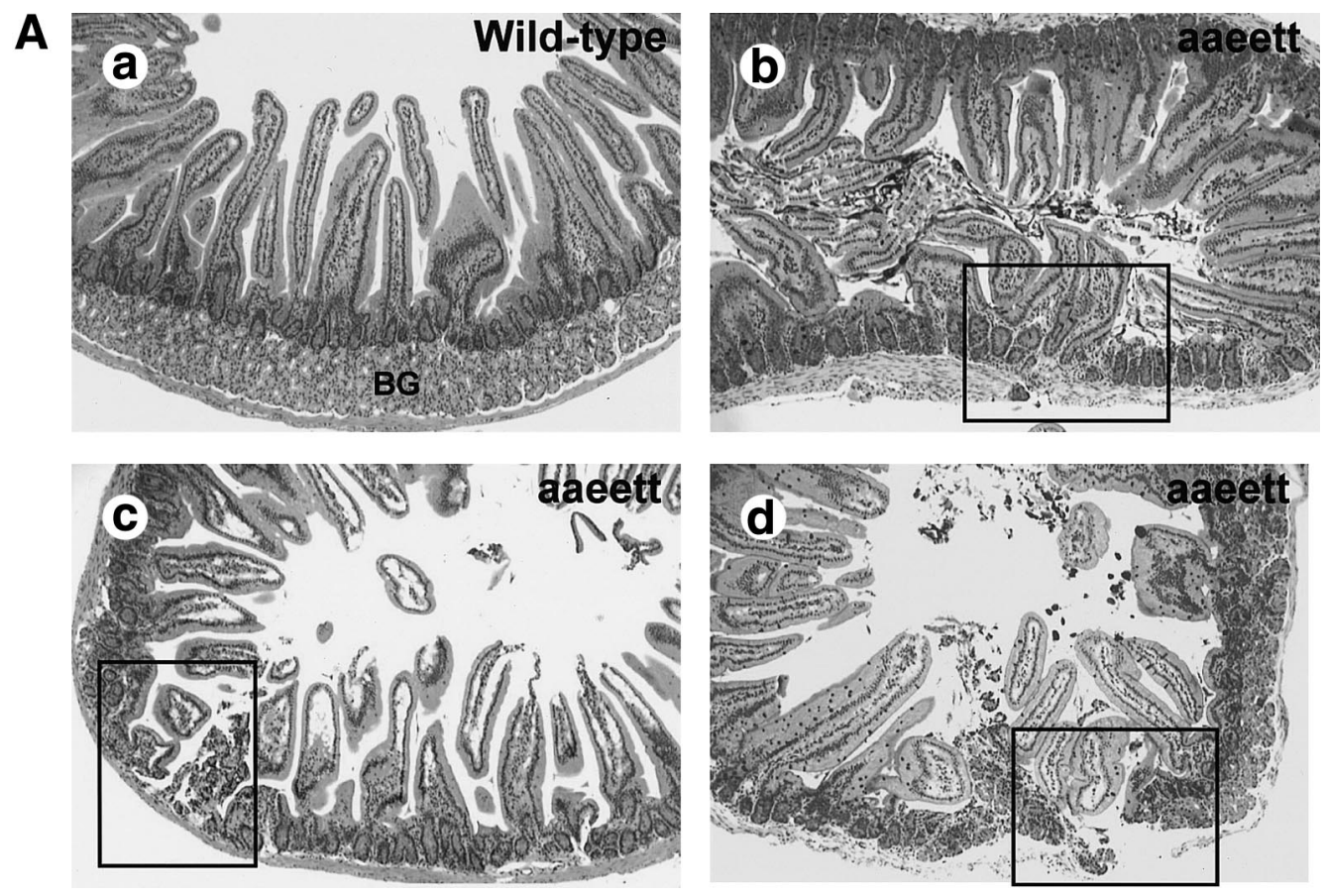

Figure 2. Morphology of wild-type and aaeett duodenum. (A) Sections of duodenum from 3-week-old wild-type (a) and aaeett (b-d) mice stained with H\&E. Spontaneous lesions (boxed) in specimens from triple null weanlings: (b) erosion, (c) ulcer, and (d) perforation. Note that while not present in $(B-D)$, Brunner's glands (BG) were observed in other aaeett duodenum sections. Original magnification 40×. (B) [see next page] Sections of duodenum were treated with Mayer's mucicarmine, which stains nuclei black and mucins pink. Note the decreased number and size of mucin positive cells (arrowheads) within the aaeett villi. Original magnification 200x.
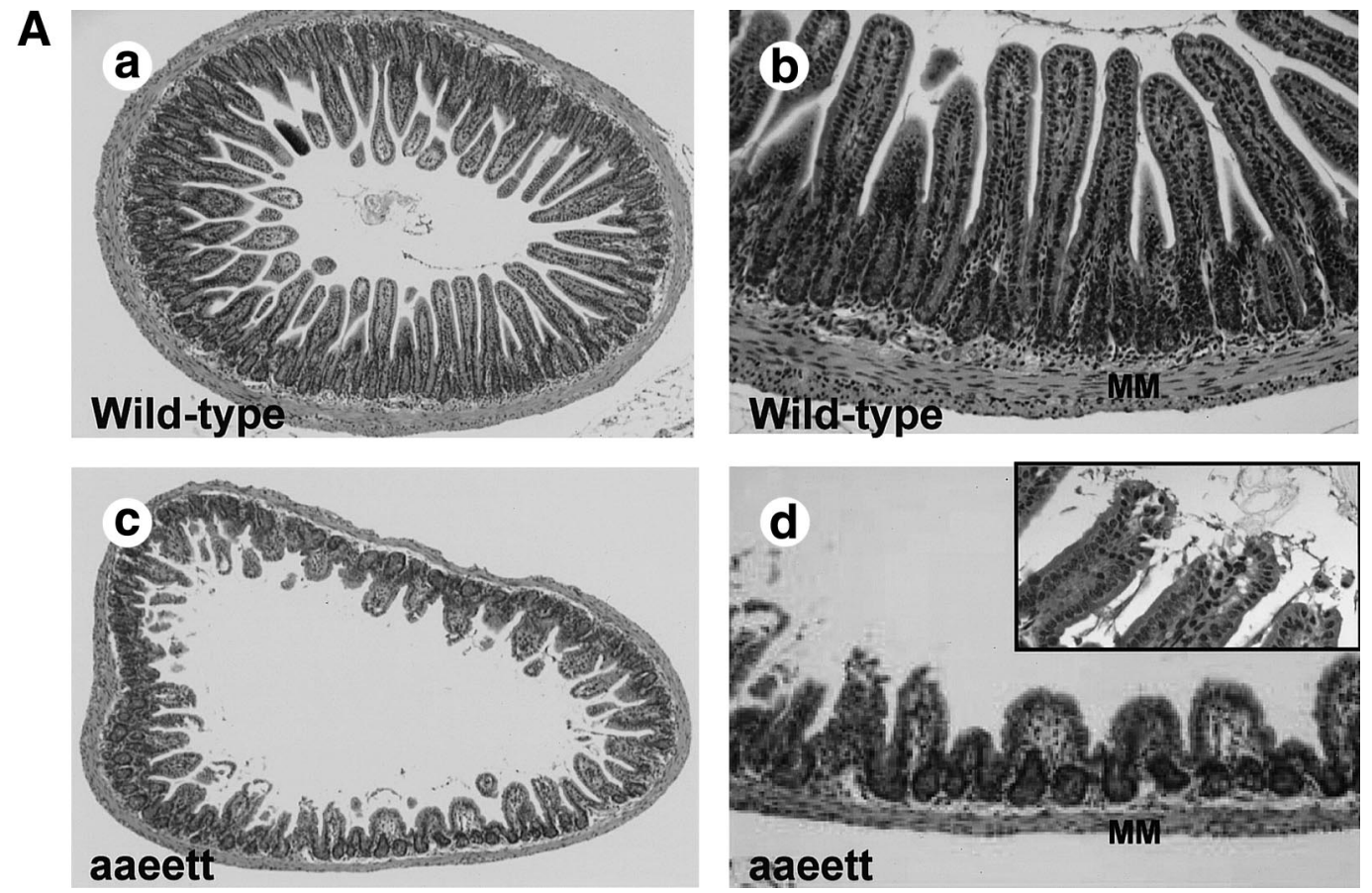

Figure 3. Morphology of wild-type and aaeett ileum. (A) Sections of ileum from 3-week-old (a, b) wild-type and (c, d) aaeett mice were stained with H\&E and viewed at low $(40 \times ; a, c)$ or high $(100 \times ; b, d)$ magnification. Note that in comparison to those of wild-type ileum, the villi in aaeett ileum appear stunted with ragged tips (inset). Also, note the reduced thickness of the muscularis mucosa layer in aaeett ileum. $(B)$ [see next page] Mice were injected intraperitoneally 1 hour before death with $50 \mathrm{mg} / \mathrm{kg}$ BrdU solution (Amersham Pharmacia Biotech, San Francisco, CA). BrdU incorporation in the ileum was visualized using BrdU antibody (Dako, New South Wales, Australia) and the ABC staining kit (Santa Cruz, Santa Cruz, CA). Results from 4 3-week-old (a, b) wild-type or (c, d) aaeett mice are shown. Note that BrdU-positive cells (examples indicated by arrowheads) are largely restricted to the crypts, with fewer positive cells detected in aaeett ileum. Original magnification $100 \times$. 
B
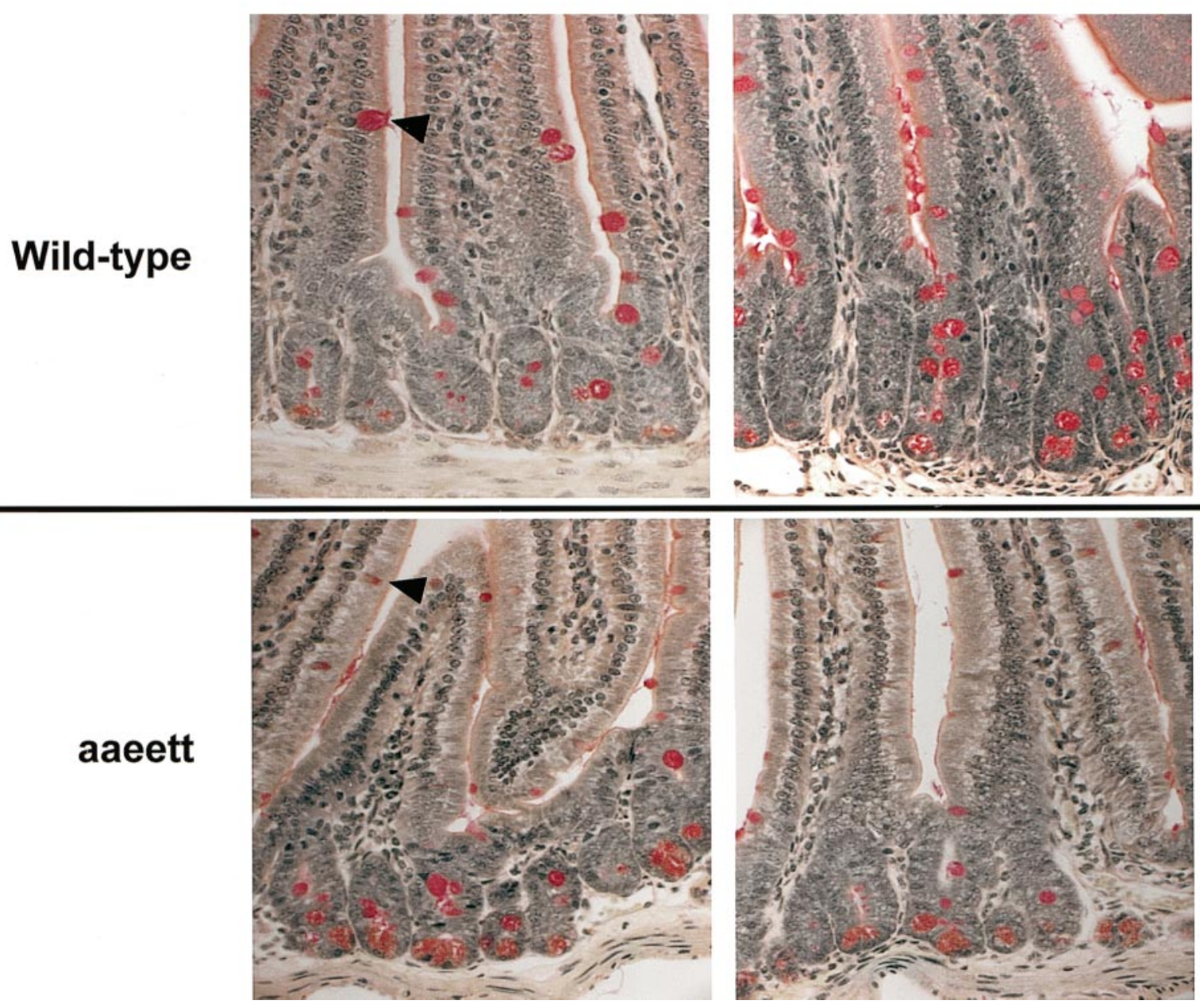

Figure 2B.

B

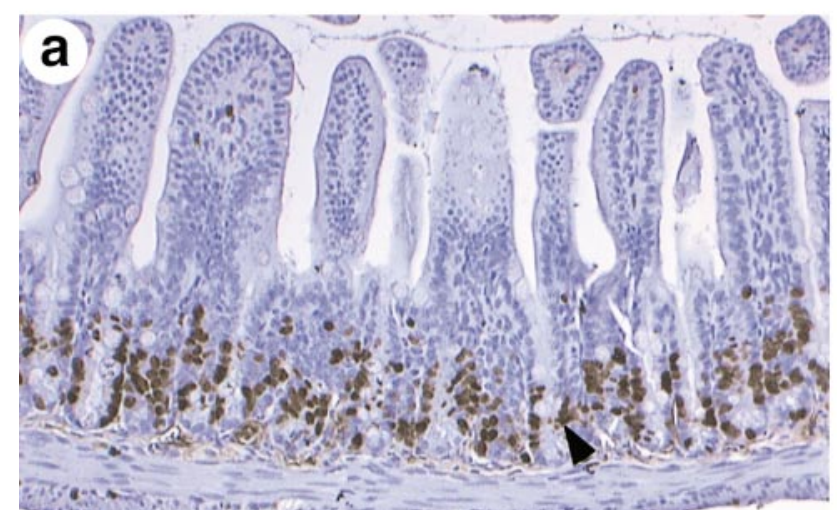

Wild-type

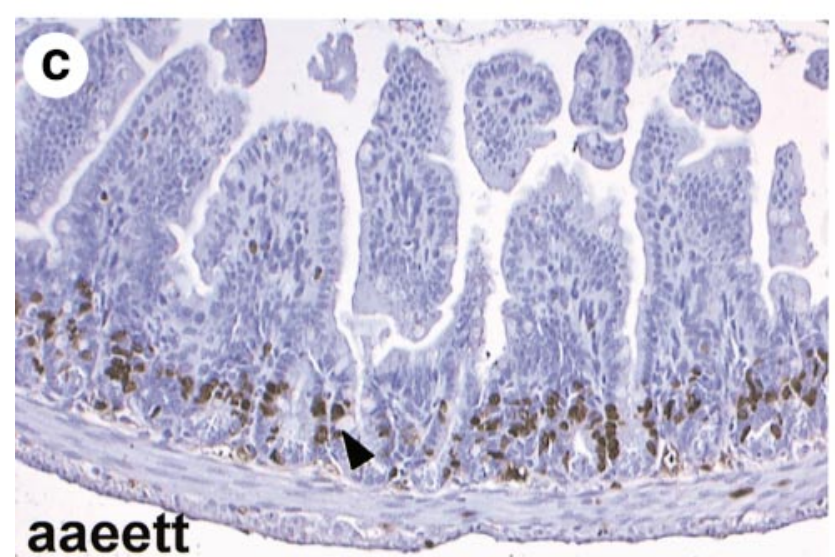

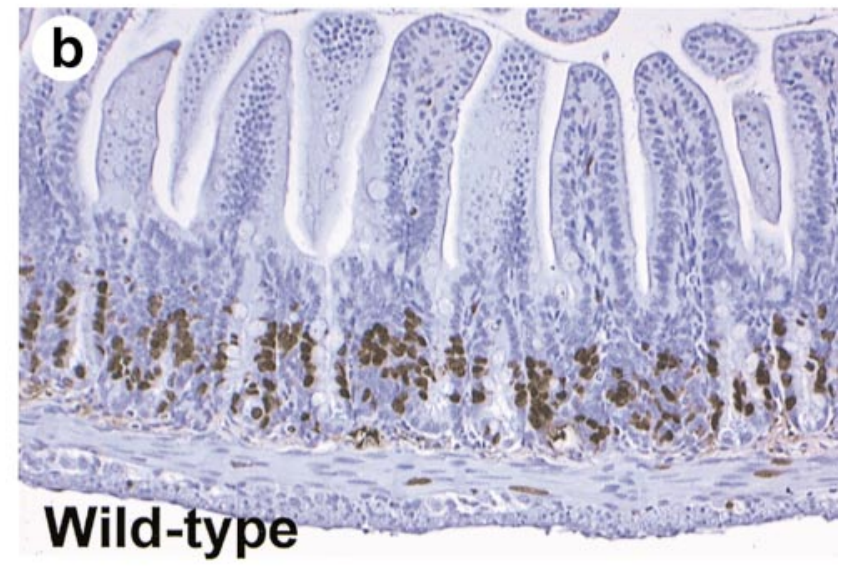

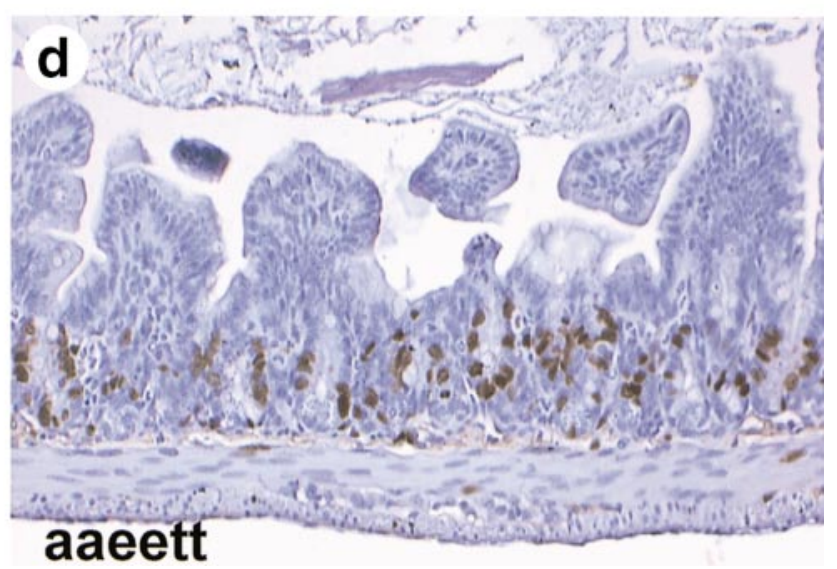


noblotted using a distinct antibody. The EGF-R protein band was confirmed by showing that it comigrated with (1) the prominent receptor band in samples of A431 cells and (2) a phosphotyrosine-containing protein detected sequentially by antiphosphotyrosine, 4G10 (Upstate Biotechnologies, Lake Placid, NY; data not shown). Figure 6 shows that EGF-R protein levels increased in both the proximal and distal halves of the small intestine of wild-type mice from none to barely detectable levels at day 14 , to readily detectable levels by day 21 . On the other hand, EGF-R levels were generally higher in the colon, and were detectable at day 2. They nevertheless increased from this time point to near maximal levels by day 7 . These results agree closely with previous detection of EGF-R in the rat small intestine by Western blot ${ }^{38}$ and in the rodent gut by binding of radiolabeled ligand. 39,40

Interestingly, the ontogeny of EGF-R protein levels in the aaeett small intestine was the mirror image of that in wild-type tissue. Thus, EGF-R protein was consistently detected at day 2 in proximal and especially distal portions of the aaeett small intestine, but then declined thereafter and was generally nondetectable by day 21 (Figure 6). Increased early receptor levels could be caused by diminished receptor down-regulation in the aaeett intestine, although an interesting possibility is that EGF-R is up-regulated as part of a compensatory response to loss of 3 of its ligands. In contrast, the time course of EGF-R expression in the aaeett colon closely matched that in the wild-type tissue. The deficiency of EGF-R in weanling aaeett small intestine is intriguing; it may be explained by a loss of ligand-dependent autocrine loops that normally induce receptor expression. ${ }^{7}$ In light of the EGF-R phenotype, it raises the question as to why more severe phenotypes are not observed in the 3-week-old aaeett intestine.

\section{Enhanced Susceptibility of EGF-Null Duodenum to Cysteamine-Induced Lesions, but Not to Dextran Sulfate Sodium-Induced Colitis}

As noted above, weanling aaeett duodenum is susceptible to spontaneous erosion and ulceration. We pursued these results by examining the role of EGF in intestinal cytoprotection because previous reports have described protective roles for EGF in various models of experimentally induced ulceration. ${ }^{13}$ EGF null mice (AAeeTT) were treated with the ulcerogen cysteamine, which selectively acts in the duodenum and has been shown to induce Brunner's glands to deplete their mucus and EGF stores. ${ }^{41,42}$ Preliminary experiments established an effective but generally nonlethal dose of cysteamine that typically induced a single lesion per mouse.

Groups of fasted adult AAeeTT and wild-type mice dosed by cysteamine gavage $(500 \mathrm{mg} / \mathrm{kg}$ ) were killed 24 or 48 hours later, and duodenal lesions scored both grossly and histologically as either erythremas, erosions, shallow/focal ulcers, ulcers, or perforations. At both time points, cysteamine treatment produced more severe lesions (either ulcers or perforations) in AAeeTT mice (Figure 7). Thus at 24 hours, 25 of 33 AAeeTT mice suffered ulcers or perforations, whereas only 12 of 32 wild-type mice had lesions of this severity $(P=0.0018)$. This trend was also present at 48 hours, although not statistically significant. These results, reproduced in 6 pilot experiments, provide important genetic evidence for a cytoprotective role for EGF in the adult duodenum.

We also examined the susceptibility of 6-month-old male aaeett colons to colitis induced by dextran sulfate sodium (DSS) administered ad libitum (1\% or $3 \%$ in drinking water for 7 days). At both doses, no significant differences were observed between the 2 genotypes $(\mathrm{n}=$

Table 1. Body Weights and Intestinal Parameters From Crossfostered Mice at 3 Weeks of Age

\begin{tabular}{|c|c|c|c|c|c|}
\hline Genotype & $\begin{array}{l}\text { Body wt } \\
(g)\end{array}$ & $\begin{array}{l}\text { Small intestine } \\
\text { wt (mg) }\end{array}$ & $\begin{array}{l}\text { Small intestine } \\
\text { length }(\mathrm{cm})^{a}\end{array}$ & $\begin{array}{l}\text { Wt:length } \\
(\mathrm{mg} / \mathrm{cm})\end{array}$ & $\begin{array}{c}\text { Number with reduced } \\
\text { villus height }{ }^{b, c}\end{array}$ \\
\hline Wild-type pups:wild-type dams $(\mathrm{n}=11)$ & $9.6 \pm 0.3$ & $528 \pm 44$ & $27.6 \pm 0.7$ & 19 & $0 / 9$ \\
\hline aaeett pups:aaeett dams $(n=16)$ & $5.8 \pm 0.3$ & $287 \pm 18$ & $22.7 \pm 0.6$ & 12 & $6 / 12$ \\
\hline aaeett pups:wild-type dams $(n=11)$ & $6.7 \pm 0.4$ & $362 \pm 28$ & $24.4 \pm 0.7$ & 14 & $3 / 9$ \\
\hline
\end{tabular}

NOTE. Crossfostering was performed as described in Results, and data were collected at 3 weeks of age.

asmall intestine was removed from pyloric to cecal junctions and contents flushed with phosphate-buffered saline. Lengths were determined on extended small intestines.

${ }^{b}$ Villus height measurements were performed using light microscopy and computer-assisted morphometry and were considered reduced if the average height of 20 well-oriented villi was at least 30\% smaller than wild-type values. Histologic assessment was performed on a subset of animals in each case.

cThe difference in the number of mice with reduced villus height, comparing aaeett pups:aaeett dams and wild-type pups:wild-type dams, was statistically significant $(P=0.0018)$. However, there was no significant difference between aaeett pups:aaeett dams and aaeett pups:wild-type dams $(P=0.6605)$. 

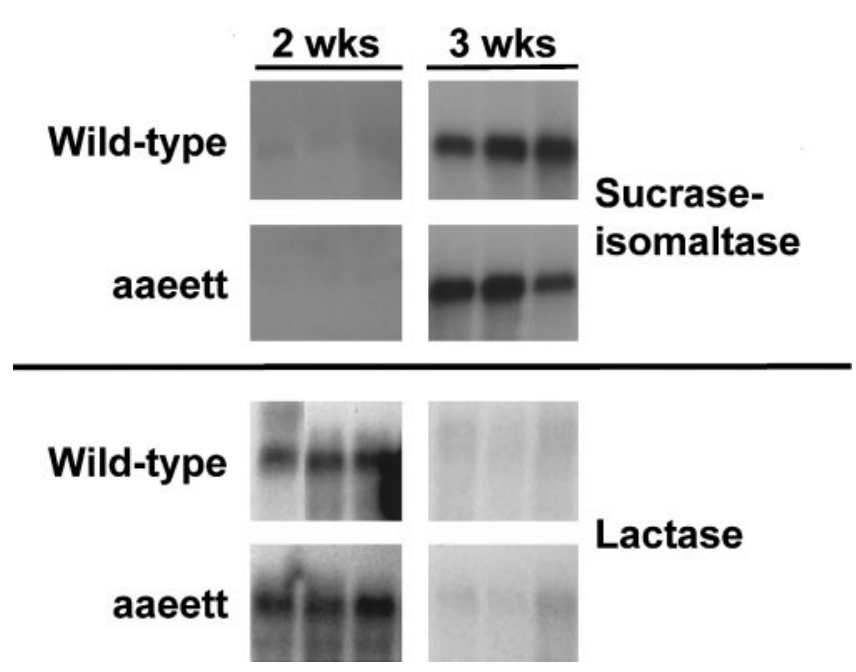

Figure 4. Expression of digestive enzyme RNAs. Samples (15 $\mu \mathrm{g})$ of total jejunal RNA from independent wild-type or aaeett mice of the indicated ages were resolved through 1\% agarose-formaldehyde gels and examined by Northern blot using cloned rodent cDNA probes for sucrase-isomaltase or lactase as described in Materials and Methods. Even loading was verified by methylene blue staining of the $18 \mathrm{~s}$ and 28 s ribosomal RNA bands. Transcript sizes are 6.8 kilobase for lactase and 6.5 kilobase for sucrase-isomaltase.

6 each) from day 1 through 7 with regard to body weight loss, stool consistency, or hemoccult assay (data not shown). Moreover, disease activity index scores ${ }^{43}$ revealed no statistically significant differences between the 2 groups at either dose.

\section{Discussion}

The phenotypes described here provides genetic proof of important roles for EGF, TGF- $\alpha$, and AR in gastrointestinal development and homeostasis. These defects, namely spontaneous ulceration and shorter, more fragile villi, are reminiscent of those of newborn EGF-R null mice in a Swiss-Webster line. ${ }^{21}$ However, they differ in their restriction to specific segments of the gut and in their amelioration with age in survivors. (Note that the majority of aaeett pups born to uniparous aaeett dams die within 1-2 days of birth; the survival rate was higher in the present study because of preselection of nursingcompetent dams.) Whereas EGF-R loss results in a necrotizing enterocolitis-like phenotype with eventual disintegration of the intestine in 1-3-week-old animals and death from wasting, in the present study most triple null mice survived to adulthood with relatively mild growth retardation. Although it remains to be established whether intestinal disintegration in EGF-R knockout mice is a primary consequence of receptor loss within the gut, these differences are nevertheless consistent with redundant roles for EGF-R ligands and with our finding that transcripts encoding all 6 EGF family members can be detected throughout the gut in broad, overlapping patterns. Collectively, these results suggest that although previous studies have focused primarily on EGF and TGF- $\alpha$, other family members are likely to play important roles in the gut. Finally, it is worth noting that no or only mild gastrointestinal defects were observed, despite progressive neonatal wasting in other lines of EGF-R null mice. ${ }^{19,20}$ This could be caused by differential activation of complementary signaling pathways in other genetic backgrounds.

As noted, triple null mice are growth impaired relative to wild-type counterparts. The difference is most severe at weaning, when the average body weight of aaeett animals is reduced by $40 \%$, and aaeett weanlings display higher-than-normal mortality (data not shown). Triple null mice remain smaller as mature adults, weighing approximately $85 \%$ that of wild-type mice. The basis for this growth impairment is unknown, but a possible explanation consistent with the phenotypes of aaeett and especially EGF-R null ${ }^{21}$ mice is that lack of endogenous neonatal growth factors leads to inferior gut development, resulting in malabsorption of nutrients and malnutrition. Alternatively, reduced quantity or quality of

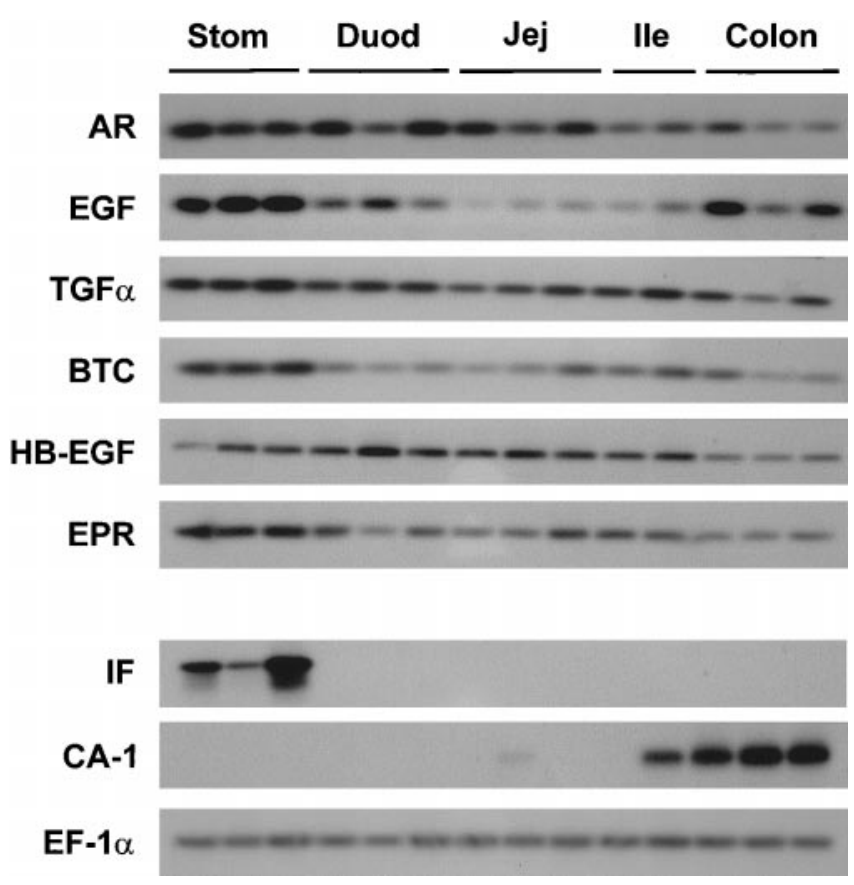

Figure 5. Expression of EGF family genes in the mouse gastrointestinal tract. Total RNA was prepared independently from the indicated tissues of multiple 3-week-old wild-type mice and was used to prepare cDNA. PCR amplification was performed with gene-specific primers after normalizing the CDNA input to yield similar levels of elongation factor (EF)- $1 \alpha$ product in all samples. Amplified products, resolved on $2 \%$ agarose gels, were visualized by Southern blotting using cDNA probes, except for carbonic anhydrase (CA)-1 and intrinsic factor (IF), which were detected using internal oligonucleotide probes. Sizes of amplified products ranged from 200 to 450 bases. 


\section{EGF-R Protein}

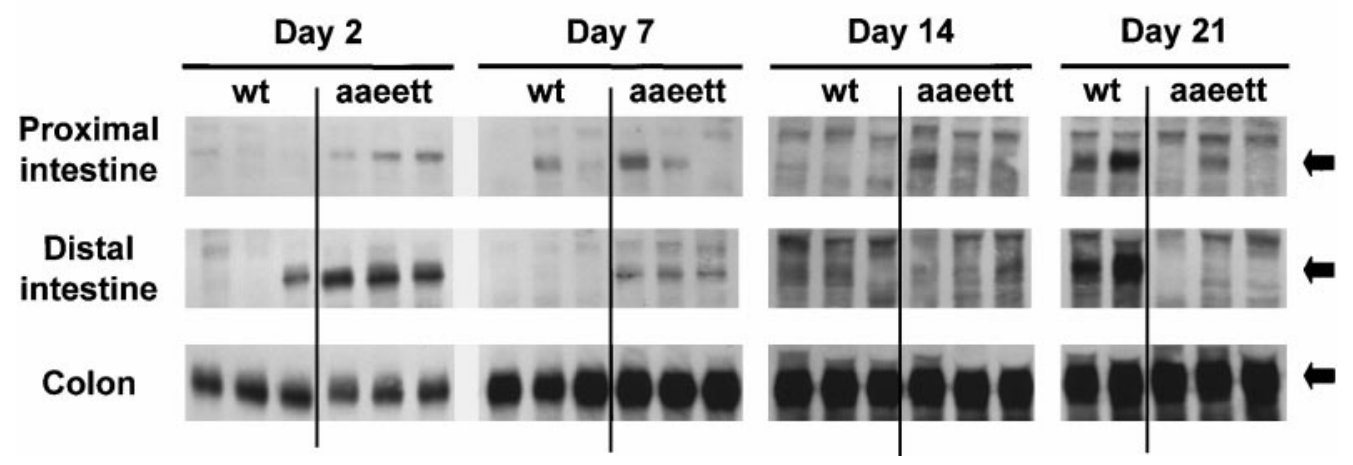

Figure 6. EGF-R protein expression during postnatal gut development. The proximal and distal halves of the small intestine and the full-length colon were harvested from wild-type (wt) or aaeett mice of the designated ages and total protein prepared as described in Materials and Methods. Aliquots $(500 \mu \mathrm{g})$ of total protein were immunoprecipitated with EGF-R antibody ERCT (H. Shelton Earp, University of North Carolina at Chapel Hill), resolved by sodium dodecyl sulfate polyacrylamide gel electrophoresis, and immunoblotted with EGF-R antibody 1005 (Santa Cruz) as described. ${ }^{25}$ Exposure times were 1-3 minutes. The position of EGF-R protein is indicated (arrows).

milk, or a deficiency of EGF family members in milk, could affect both gut development and pup nourishment. The results of our crossfostering experiments address both these possibilities. Thus, optimal pup growth required both maternal and neonatal sources of growth factors. Because in our crossfostering experiments, the observed defects in ileal morphology were principally correlated with the lack of endogenous neonatal growth factors, the precise connection between milk-borne ligands and pup growth is unclear. Of course, it is possible that these milk-derived growth factors influence intestinal development or function without affecting gross architecture. Alternatively, it has been previously suggested that milk-derived growth factor can be absorbed into the circulation. ${ }^{44}$

Interestingly, the histologic defects described here were observed at 3 weeks of age, but not at postnatal days 2,7 , and 14, a time course that agrees with the peak induction of EGF-R in the small intestine (Figure 6). This could indicate that EGF family members have an important role in the gut during the transition from milk to solid food, although we observed no effect on the change in relevant digestive enzyme profiles. Perhaps more likely is that EGF family members are required for the dramatic increase in crypt cell proliferation during the third postnatal week that results in a significant rise in villus height. ${ }^{45}$ Supporting this, villus length and crypt cell proliferation were both reduced in the 3-weekold ileum of triple null mice. The fact that truncated villi were observed only in the ileum may indicate a specific requirement for these particular EGF-R ligands in this segment of the gut, although the respective transcripts were not selectively expressed in this compartment (Figure 5). Alternatively, because the onset of enhanced crypt cell proliferation varies along the proximal-to-distal axis, failure to detect delayed villus elongation in other segments may simply be a consequence of the time points analyzed. Villi in the ileum of mature mice seemed normal, indicating that the defect resulting from loss of $\mathrm{EGF} / \mathrm{TGF}-\alpha / \mathrm{AR}$ reflects a delay in villus extension, rather than a permanent condition. Conceivably, this recovery is caused by activities of remaining EGF family members, although we did not observe up-regulation of the remaining EGF-R ligands.

We observed no obvious phenotype in the adult gut arising from the loss of the respective EGF family members, but our results indicate an impaired cytoprotective response in both neonatal and adult duodenum. We detected spontaneous ulcers in roughly half of triple null weanling samples and aggravated injury from cysteamine treatment in adult mice lacking EGF alone. These results extend numerous reports of pharmacologic roles for EGF family members (particularly EGF) in maintenance of mucosal integrity by confirming a physiologic requirement for these ligands. On the other hand, susceptibility of the adult aaeett colon to DSS-induced colitis was not affected by loss of the 3 ligands, a result that contrasts with reports of increased susceptibility of TGF- $\alpha$ knockout and waved-2 mice by Egger et al. ${ }^{23,46}$ This apparent discrepancy has several possible explanations. The TGF- $\alpha$ knockout mice used previously were maintained on a B6 background, whereas our mice are mixed B6/ 129. Additionally, we used older mice of opposite sex (male). Susceptibility to DSS varied with background strain and was more severe in male mice. ${ }^{47}$ Consistent with these results, the $5 \%$ DSS dose used by Egger et al. was lethal to our male aaeett and wild-type mice. Hence, the enhanced susceptibility of our mice may have masked 

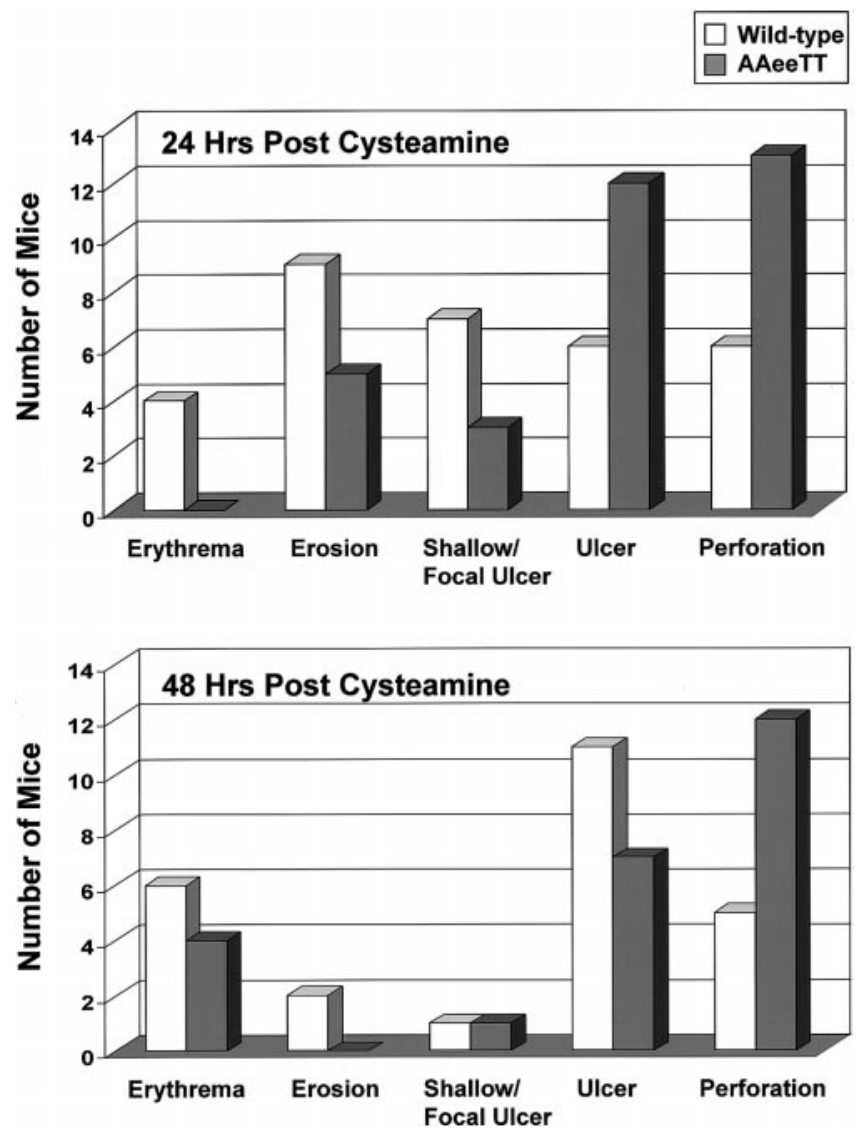

Figure 7. Cysteamine-induced duodenal lesions in adult wild-type and EGF-null (AAeeTT) mice. Mice were administered cysteamine by gavage, and killed 24 or 48 hours later. Lesions were characterized as follows: erosion, reduction in villus height or integrity but mucosal layer intact; shallow/focal ulcer, microscopic breach in mucosal layer with minimal interruption in epithelial basement membrane; ulcer, obvious interruption and injury of mucosal layer, with damage and inflammation extending into submucosa; and perforation, penetration of full thickness of intestinal wall, including interruption in muscularis layers. Open bars, wild-type; solid bars, AAeeTT. Note that at both time points, EGF null mice displayed more severe lesions (either ulcers or perforations) in response to cysteamine treatment, although the differences were statistically significant only at 24 hours.

previously observed differences between the genotypes. A more severe phenotype in waved-2 mice could be attributable to the fact that the associated EGF-R defect would eliminate signaling responses to all EGF family ligands, and additionally impair activation of related ERBB proteins arising from receptor heterodimerization.

The appearance of spontaneous lesions in the duodenum of aaeett mice is likely related to the aberrant mucin staining and reduced ITF protein expression observed in this tissue. The fact that spontaneous lesions were present only in the duodenum of aaeett mice is consistent with observations that mice treated with an EGF-R inhibitor develop lesions only in the duodenum. ${ }^{48}$ It may also reflect the importance of EGF-producing Brunner's glands in this segment of the intestine and/or may be linked to our observation that several EGF family genes are preferentially expressed in stomach (Figure 5). Growth factors produced in the stomach could be released with chyme, where they would be available to signal to the basolateral EGF-R in the event of duodenum mucosal damage. The temporal restriction of spontaneous lesions to weaning age may reflect a natural propensity of the duodenum to ulceration at this time, perhaps related to shifts in diet and gastric or pancreatic secretions. Alternatively, it could reflect reparative activities of the remaining ligands, BTC, HB-EGF, and EPR, in more distal segments of the intestine. This and related issues will be the subject of future investigation.

\section{References}

1. Lee DC, Fenton EF, Berkowitz EA, Hissong MA. Transforming growth factor- $\alpha$ : expression, regulation and biological activities. Pharm Rev 1995; 47:51-85.

2. Riese DJ, Stern DF. Specificity within the EGF family/ErbB receptor family signaling network. BioEssays 1998;20:41-48.

3. Beerli RR, Hynes NE. Epidermal growth factor-related peptides activate distinct subsets of ERBB receptors and differ in their biological activities. J Biol Chem 1996;271:6071-6076.

4. Shoyab M, Plowman GD, McDonald VL, Bradley JG, Todaro GJ. Structure and function of human amphiregulin: a member of the EGF family. Science 1989;243:1074-1076.

5. Ebner R, Derynck R. Epidermal growth factor and transforming growth factor-alpha: differential intracellular routing and processing of ligand-receptor complexes. Cell Regul 1991;2:599-612.

6. Carpenter G. Epidermal growth factor is a major growth-promoting agent in human milk. Science 1980;210:198-199.

7. Barnard JA, Beauchamp RD, Russell WE, Dubois RN, Coffey RJ. Epidermal growth factor-related peptides and their relevance to gastrointestinal pathophysiology. Gastroenterology 1995;108: $564-580$

8. Berseth CL. Enhancement of intestinal growth in neonatal rats by epidermal growth factor in milk. Am J Physiol 1987;253:G662G665.

9. Koldovsky O. The potential physiological significance of milkborne hormonally active substances for the neonate. J Mammary Gland Biol Neoplasia 1996;1:317-322.

10. Xu R-J. Development of the newborn Gl tract and its relation to colostrum/milk intake: a review. Reprod Fertil Dev 1996;8: 35-48.

11. Sandgren EP, Luetteke NC, Palmiter RD, Brinster RL, Lee DC. Overexpression of TGF alpha in transgenic mice: induction of epithelial hyperplasia, pancreatic metaplasia, and carcinoma of the breast. Cell 1990;61:1121-1135.

12. Henning SJ, Rubin DC, Shulman RJ. Ontogeny of the intestinal mucosa. In: Johnson RL, ed. Physiology of the gastrointestinal tract. 3rd ed. New York: Raven, 1994:571-610.

13. Uribe JM, Barrett KE. Nonmitogenic actions of growth factors: an integrated view of their role in intestinal physiology and pathophysiology. Gastroenterology 1997;112:255-268.

14. Jones MK, Tomikawa M, Mohajer B, Tarnawski AS. Gastrointestinal mucosal regeneration: role of growth factors. Front Biosci 1999;4:d303-d309.

15. Cook GA, Yeomans ND, Giraud AS. Temporal expression of trefoil peptides in the TGF- $\alpha$ knockout mouse after gastric ulceration. Am J Physiol 1997;272:G1540-G1549.

16. Luetteke NC, Qiu TH, Peiffer RL, Oliver P, Smithies O, Lee DC. TGF $\alpha$-deficiency results in hair follicle and eye abnormalities in targeted and waved-1 mice. Cell 1993;73:263-278. 
17. Mann GB, Fowler KJ, Gabriel A, Nice EC, Williams RL, Dunn AR. Mice with a null mutation of the TGF- $\alpha$ gene have abnormal skin architecture, wavy hair, and curly whiskers and often develop corneal inflammation. Cell 1993;73:249-261.

18. Luetteke NC, Qiu TH, Fenton SE, Troyer KL, Riedel RF, Chang A, Lee DC. Targeted inactivation of the EGF and amphiregulin genes reveals distinct roles for EGF receptor ligands in mouse mammary gland development. Development 1999;126:2739-2750.

19. Sibilia M, Wagner EF. Strain-dependent epithelial defects in mice lacking the EGF receptor. Science 1995;269:234-237.

20. Threadgill DW, Dlugosz AA, Hansen LA, Tennenbaum T, Lichti U, Yee D, LaMantia C, Mourton T, Herrup K, Harris RC, Barnard JA, Yuspa SH, Coffey RJ, Magnuson T. Targeted disruption of mouse EGF receptor: effect of genetic background on mutant phenotype. Science 1995;269:230-233.

21. Miettinen PJ, Berger JE, Maneses J, Phung Y, Pedersen RA, Werb Z, Derynck R. Epithelial immaturity and multiorgan failure in mice lacking epidermal growth factor receptor. Nature 1995;376:337341.

22. Luetteke NC, Phillips HK, Qiu TH, Copeland NG, Earp HS, Jenkins NA, Lee DC. The mouse waved-2 phenotype results from a point mutation in the EGF receptor tyrosine kinase. Genes Dev 1994; 8:399-413.

23. Egger B, Procaccino F, Lakshmanan J, Reinshagen M, Hoffmann P, Patel A, Reuben W, Gnanakkan S, Liu L, Barajas L, Eysselein VE. Mice lacking transforming growth factor alpha have an increased susceptibility to dextran sulfate-induced colitis. Gastroenterology 1997;113:825-832.

24. Helmrath MA, Erwin CR, Warner BW. A defective EGF-receptor in waved-2 mice attenuates intestinal adaptation. J Surg Res 1997; 69:76-80.

25. Schroeder JA, Lee DC. Dynamic expression and activation of ERBB receptors in the developing mouse mammary gland. Cell Growth Diff 1998;9:451-464.

26. Büller HA, Kothe MJC, Goldman DA, Grubman SA, Sasak WV, Matsudaira PT, Montgomery RK, Grand RJ. Coordinate expression of lactate-phlorizin hydrolase mRNA and enzyme levels in rat intestine during development. J Biol Chem 1990;265:69786983.

27. Romano M, Polk WH, Awad JA, Arteaga CL, Nanney LB, Wargovich MJ, Kraus ER, Boland CR, Coffey RJ. Transforming growth factor $\alpha$ protection against drug-induced injury to the rat gasric mucosa in vivo. J Clin Invest 1992;90:2409-2421.

28. Cook GA, Yeomans ND, Giraud AS. Temporal expression of trefoil peptides in the TGF- $\alpha$ knockout mouse after gastric ulceration. Am J Physiol 1997;272:G1540-G1549.

29. Taupin D, Wu D-C, Jeon W-K, Devaney K, Wang TC, Podolsky DK. The trefoil gene family are coordinately expressed immediateearly genes: EGF receptor-and MAP kinase-dependent interregulation. J Clin Invest 1999;103:R31-R38.

30. Giraud AS. Lessons from genetically engineered animal models $X$. Trefoil peptide and EGF receptor/ligand transgenic mice. Am J Physiol Gastrointest Liver Physiol 2000;278:G501-G506.

31. Podolsky DK. Mechanisms of regulatory peptide action in the gastrointestinal tract: trefoil peptides. J Gastroenterol 2000;35: $69-74$.

32. Longman RJ, Douthwaite J, Sylvester PA, Poulsom R, Corfield AP, Thomas MG, Wright NA. Coordinated localisation of mucins and trefoil peptides in the ulcer associated cell lineage and the gastrointestinal mucosa. Gut 2000;47:792-800.

33. Suemori S, Lynch-Devaney K, Podolsky DK. Identification and characterization of rat intestinal trefoil factor: tissue- and cellspecific member of the trefoil protein family. Proc Natl Acad Sci U S A 1991;88:11017-11021.

34. Kellett M, Potten CS, Rew DA. A comparison of in vivo cell proliferation measurements in the intestine of mouse and man. Epithelial Cell Biol 1992;1:147-155.

35. Chandrasena G, Sunitha I, Lau C, Nanthakumar NN, Henning SH. Expression of sucrase-isomaltase-isomaltase mRNA along the villus-crypt axis in the rat small intestine. Cell Mol Biol 1992;38: 243-254.

36. Maeda M, Asahara S, Nishi T, Mushiake S, Oka T, Shimada S, Chiba T, Tohyama M, Futai M. The rat intrinsic factor gene: its 5 '-upstream region and chief cell-specific transcription. J Biochem 1995;117:1305-1311.

37. Wang Y, Harvey C, Rousset M, Swallow DM. Expression of human intestinal mRNA transcripts during development: analysis by a semiquantitative RNA polymerase chain reaction method. Pediatr Res 1994;36:514-521.

38. Polk DB. Ontogenic regulation of phospholipase C-g1 activity and expression in the rat small intestine. Gastroenterology 1994; 107:109-116.

39. Menard D, Pothier P, Gallo-Payett N. Epidermal growth factor receptors during postnatal development of the mouse colon. Endocrinology 1987;121:1548-1554.

40. Gallo-Payet N, Pothier P, Hugon JS. Ontogeny of EGF receptors during postnatal development of the small intestine. J Pediatr Gastroenterol Nutr 1987;6:114-120.

41. Kirkegaard P, Olsen PS, Poulsen SS, Nexo E. Epidermal growth factor inhibits cysteamine-induced duodenal ulcers. Gastroenterology 1983;85:1277-1283.

42. Olsen PS. Role of epidermal growth factor in gastroduodenal mucosal protection. J Clin Gastroenterol 1988;10:S146-S151.

43. Cooper HS, Murthy SNS, Shah RS, Sedergran DJ. Clinicopathologic study of dextran sulfate sodium experimental murine colitis. Lab Invest 1993;69:238-249.

44. Purushotham KR, Offenmüller K, Bui AT, Zelles T, Blazsek J, Schultz GS, Humphreys-Beher G. Absorption of epidermal growth factor occurs through the gastrointestinal tract and oral cavity in adult rats. Am J Physiol 1995;269:G867-G873.

45. Gordon JI, Hermiston ML. Differentiation and self-renewal in the mouse gastrointestinal epithelium. Curr Opin Cell Biol 1994;6: 795-803.

46. Egger B, Buchler MW, Lakshmanan J, Moore P, Eysselein VE. Mice harboring a defective epidermal growth factor receptor (waved-2) have an increased susceptibility to acute dextran sulfate-induced colitis. Scand J Gastroenterol 2000;35:1181-1187.

47. Mahler M, Bristol IJ, Leiter EH, Workman AE, Birkenmeier EH, Elson CO, Sundberg JP. Differential susceptibility of inbred mouse strains to dextran sulfate sodium-induced colitis. Am J Physiol 1998;274:G544-G551.

48. Ritland SR, Gendler SJ, Burgart LJ, Fry DW, Nelson JM, Bridges AJ, Andress L, Karnes WE. Inhibition of epidermal growth factor receptor tyrosine kinase fails to suppress adenoma formation in $\mathrm{Apc}^{\mathrm{Min}}$ mice but induces duodenal injury. Cancer Res 2000;60: 4678-4681.

Received September 22, 2000. Accepted March 14, 2001.

Address requests for reprints to: David C. Lee, Ph.D., Department of Biochemistry \& Biophysics, Campus Box 7260, School of Medicine, University of North Carolina at Chapel Hill, Chapel Hill, North Carolina 27599-7260. e-mail: dclee@med.unc.edu; fax: (919) 966-2852.

Supported by National Institutes of Health (NIH) grant CA43793 (to D.C.L.), an institutional grant from the UNC Center for Gastrointestinal Biology and Disease (to N.C.L.), an NIH postdoctoral grant CA69779 (to M.L.S.), and an NIH predoctoral training grant GM08581 (to K.L.T.).

The authors thank Drs. Susan Henning and Randy Fuller for helpful advice, Aileen Chang for genotyping, Dominic Moore for statistical analyses, and Drs. Robert Bagnell and Robert Schoonhoven for assistance with microscopic image analysis. 